Ann. Biol. anim. Bioch. Biophys., 1979, 19 (3 B), 807-812.

\title{
Variations des activités maltase et invertase dans l'intestin de poulet en fonction de l'âge et du régime alimentaire
}

J. C. BLUM, Anne GAUTHIER, S. GUILLAUMIN

Station de Recherches Avicoles, I.N.R.A., Nouzilly, 37380 Monnaie.

Summary. Variations of intestinal maltase and sucrase activities in chicks according to age and dief.

The maltase and sucrase activities of 4 and 8-week old chickens were measured in the duodenum, jejunum and ileum. Semi-synthetic diets were given from hatching. They were isoenergetic, but had different carbohydrate content : 61 p. 100 starch, 61 p. 100 sucrose, carbohydrate-free ( 35 p. 100 cellulose, 26 p. $100 \mathrm{fat}$ ). A commercial type diet was given to a control group. The chickens were fasted $14 \mathrm{hrs}$ before decapitation. Disaccharidase activities were maximal in the jejunum. The highest activities were observed with the two carbohydrate diets. Sucrose had more effect on the proximal parts of the intestine : the duodenum and jejunum. In the ileum, disaccharidase activities were greater with the starch diet. Activity in the carbohydrate-free diet was less than 50 p. 100. The commercial diets gave intermediate results between the carbohydrate and carbohydrate-free diets. The diets affected disaccharidase activities, and their influence was evident whether the enzyme activities were expressed as units per tissue protein or as units per wet weight. The response, however, was not specific. The activities of maltase and sucrase increased concomitantly. Carbohydrate stimulation tended to decrease from 4 to 8 weeks of age.

\section{Introduction.}

L'intestin joue un rôle essentiel dans les étapes ultimes de la digestion des glucides. D'une analyse bibliographique récente (Levin, 1976), il ressort que les études effectuées chez les oiseaux sont rares en regard de celles réalisées chez les mammifères. Seul Siddons (1972) a envisagé l'influence de la composition des régimes sur l'activité des disaccharidases. Mais ses mesures ont été faites à un seul âge, chez le jeune poussin de 25 jours. En outre, l'intestin grêle a été isolé dans son entier sans distinction des différentes parties : duodénum, jéjunum et iléon. Nous avons repris cette étude en cherchant à préciser l'influence de l'âge ef le rôle des glucides alimentaires sur les activités de la maltase et de l'invertase. 


\section{Matériel et méthodes.}

Les animaux ont été élevés selon le protocole indiqué précédemment (Gauthier, Larbier et Blum, 1979). Pour l'essentiel 3 lots de poulets sont alimentés chacun depuis la naissance avec un régime synthétique caractérisé par sa composition glucidique : 61 p. 100 d'amidon, 61 p. 100 de saccharose ou absence de glucides digestibles (les 61 p. 100 de glucides sont remplacés par un mélange isoénergétique de graisse et de cellulose). La partie commune des 3 régimes (49 p. 100) apporte tous les compléments indispensables à la croissance. Un quatrième régime de type «commercial » (maïssoja) est distribué d̀ un lot témoin.

Dans chacun des 4 lots, on sacrifie 10 poulets à l'âge de 4 semaines et 6 à l'âge de 8 semaines. Le sacrifice est effectué par décapitation après un court jeûne de $14 \mathrm{~h}$. L'intestin est immédiatement prélevé et séparé conventionnellement en 3 parties : l'anse duodénale autour du pancréas, le jéjunum jusqu'au diverticule de Meckel, l'iléon en amont de l'abouchement des cæca. Chaque fragment, soigneusement dégraissé, est ouvert longifudinalement ef lavé avec du sérum physiologique glacé ; il est conservé congelé jusqu'au moment du dosage.

L'extraction et les mesures d'activités enzymatiques sont effectuées selon la méthode de Dahlquist (1964) avec des modifications mineures : broyage de l'intestin (paroi et muqueuse) dans dix fois son poids de sérum physiologique; hydrolyse du maltose et du saccharose à des $\mathrm{pH}$ optimum trouvés très légèrement inférieurs pour le

\section{TABLEAU 1}

Valeurs moyennes ( \pm écart-type) des activités maltase ( $\mathrm{mg}$ de glucose libéré/g de protéines/mn)

dans choque segment de l'intestin grêle en fonction du régime consommé jusqu'aux âges de 4 et 8 semaines

\begin{tabular}{|c|c|c|c|c|}
\hline \multirow{2}{*}{ Poulets } & \multirow{2}{*}{$\begin{array}{c}\text { Régime } \\
\text { commercial }\end{array}$} & \multicolumn{3}{|c|}{ Régimes synthétiques } \\
\hline & & Amidon & Saccharose & Graisse-cell. \\
\hline \multicolumn{5}{|l|}{4 semaines } \\
\hline $\begin{array}{l}\text { duodénum ........ } \\
\text { jéjunum ......... } \\
\text { lléon } \ldots \ldots \ldots \ldots\end{array}$ & $\begin{array}{l}16,2\left({ }^{d *}\right) \\
\pm 2,3 \\
22,3(b c) \\
\pm 1,4 \\
14,9(d e) \\
\pm 2,2\end{array}$ & $\begin{array}{c}16,5(d) \\
+1,9 \\
25,9(a b) \\
\pm 2,2 \\
16,0(d) \\
\pm 1,1\end{array}$ & $\begin{array}{c}22,5\left(^{b}\right) \\
\pm 2,0 \\
27,6(a) \\
\pm 2,2 \\
11,4\left(^{(e f}\right) \\
\pm 1,3\end{array}$ & $\begin{array}{l}8,4(f) \\
\pm 0,7 \\
12,8\left({ }^{e}\right) \\
\pm 0,9 \\
9,5(f) \\
\pm 0,7\end{array}$ \\
\hline \multicolumn{5}{|l|}{8 semaines } \\
\hline $\begin{array}{l}\text { duodénum } \ldots \ldots \ldots \\
\text { jéjunum } \ldots \ldots \ldots \ldots \\
\text { Iléon } \ldots \ldots \ldots \ldots\end{array}$ & $\begin{aligned} & 13,0(\mathrm{de}) \\
\pm & 2,0 \\
& 19,7(\mathrm{c}) \\
\pm & 2,6 \\
& 11,9(\mathrm{ef}) \\
\pm & 1,3\end{aligned}$ & $\begin{array}{r}15,6(d) \\
\pm 1,3 \\
20,2(c) \\
\pm 1,5 \\
13,4(d e) \\
\pm 1,3\end{array}$ & $\begin{array}{r}15,4\left(^{d}\right) \\
+2,1 \\
23,4\left(^{b}\right) \\
\pm 1,6 \\
6,0\left(^{g}\right) \\
+1,4\end{array}$ & $\begin{aligned} & 9,6(f) \\
\pm & 1,0 \\
& 14,6(d e) \\
\pm & 1,5 \\
& 6,8(f g) \\
\pm & 1,2\end{aligned}$ \\
\hline
\end{tabular}

* Les valeurs qui ne sont pas suivies de la même lettre diffèrent significativement entre elles $(P<0,05)$. 
poulet (respectivement 5,85 ef 6,05 contre 6 et 6,2 recommandés pour l'homme) ; dosage du glucose par la glucose-oxydase à l'aide de l'auto-analyseur Technicon. Pour l'expression des résultats, le poids frais de l'intestin apparaissait criticable du fait de la variabilité d'épaisseur de la paroi. On a dosé les protéines totales, extraites avec les enzymes, par la méthode de Lowry et al. (1951) adaptée à l'auto-analyseur Technicon. La signification statistique des résultats a été établie par analyse de variance.

\section{Résultats.}

Nous avons exprimé ces activités disaccharidases par gramme de protéines. Non retenue dans cette courte note, l'expression par gramme frais conduit à des résultats semblables : différence entre segments intestinaux atténuée; effel de l'âge augmenté.

A) Activités maltase (tabl. 1).

A un âge donné ( 4 ou 8 semaines) il existe des différences d'activités d'un segment à l'autre. D'une manière générale les activités les plus fortes sont observées dans le jéjunum.

La composition du régime a une influence variable au long de l'intestin. Avec le régime privé de glucides (graisse-cellulose), les différences entre segments intestinaux sont relativement faibles. Un apport glucidique (amidon ou saccharose) augmente considérablement les activités maltase. Avec le saccharose l'augmentation intéresse surtout les parties proximales : l'activité du duodénum est presque triplée, celle du jéjunum est multipliée par deux, tandis que celle de l'iléon n'est que faiblement accrue. Avec l'amidon l'augmentation est du même ordre, mais elle intéresse davantage les parties distales : tout au long du tractus l'activité est presque régulièrement multipliée par 2 lorsque le régime gras est pris comme référence. Si l'on compare l'action des deux sucres, on conclut à une stimulation des activités maltase plus forte par le saccharose dans le duodénum et par l'amidon dans l'iléon ; tandis que dans le jéjunum la stimulation est identique. L'influence du régime commercial, qui renferme beaucoup de maïs ef un peu de suif, est liée à sa composition. Les valeurs trouvées ne sont pas différentes de celles obtenues avec le régime amidon en demeurant quelque peu inférieures (analogie avec le régime gras).

Il existe un effet significatif de l'âge qui dépend de la nature des régimes. Avec le régime gras, sans glucides, l'activité maltase ne varie que faiblement de 4 à 8 semaines. On observe surtout une différenciation accrue entre les 3 segments : activité du jéjunum relativement plus forte en regard de l'iléon. Avec les régimes glucidiques on observe une diminution particulièrement sensible dans le cas du saccharose. Le régime commercial a, là encore, une influence infermédiaire entre régimes gras et amidon.

\section{B) Activité invertase (tabl. 2).}

Nombre de constatations faites en analysant les mesures d'activité maltase peuvent être reprises à propos de l'invertase.

L'activité est maximum dans le jéjunum. La composition du régime a une influence. La stimulation induite par les régimes glucidiques affecte inégalement le tractus intes- 
tinal et exacerbe les différences entre segments. Avec le saccharose les activités sont accrues dans la partie proximale; avec l'amidon la stimulation est moins marquée dans le duodénum, mais elle s'étend jusqu'à l'iléon. Le régime commercial a, comme précédemment, une influence intermédiaire liée à sa composition : forte teneur en amidon, présence de graisse.

Il existe un effet significatif de l'âge, qui dépend du régime. On observe des diminutions d'activité dans le seul cas des régimes riches en glucides.

TABLEAU 2

Valeurs moyennes ( \pm écart-fype) des activités invertase

( $\mathrm{mg}$ de glucose libéré/g de protéine/min)

dans chaque segment de l'intestin grêle en fonction du régime consommé jusqu'aux âges de 4 et 8 semaines

\begin{tabular}{|c|c|c|c|c|}
\hline \multirow{2}{*}{ Poulets } & \multirow{2}{*}{$\begin{array}{l}\text { Régime } \\
\text { commercial }\end{array}$} & \multicolumn{3}{|c|}{ Régimes synthétiques } \\
\hline & & Amidon & Saccharose & Graisse-Cell. \\
\hline \multicolumn{5}{|l|}{4 semoines } \\
\hline $\begin{array}{l}\text { duodénum } \ldots \ldots \ldots \\
\text { jéjunum } \ldots \ldots \ldots \ldots \\
\text { iléon } \ldots \ldots \ldots \ldots \ldots\end{array}$ & $\begin{aligned} & 4,6(g h) \\
+ & 0,9 \\
& 8,2(c d) \\
\pm & 0,8 \\
& 4,2(g h) \\
\pm & 0,8\end{aligned}$ & $\begin{aligned} & 4,8(f g) \\
& \pm 0,6 \\
& 10,1(b c) \\
& \pm 0,8 \\
& 4,9(f g) \\
& \pm 0,5\end{aligned}$ & $\begin{aligned} & 8,4(c d) \\
& \pm 1,2 \\
& 12,9(a) \\
& \pm 1,0 \\
& 3,6\left(^{(h i}\right) \\
& \pm 0,4\end{aligned}$ & $\begin{aligned} & 2,4\left(^{(j)}\right. \\
\pm & 0,3 \\
& 4,0\left(^{h}\right) \\
\pm & 0,4 \\
& 2,7\left(^{(i j)}\right. \\
\pm & 0,2\end{aligned}$ \\
\hline \multicolumn{5}{|l|}{8 semaines } \\
\hline $\begin{array}{l}\text { duodénum } \ldots \ldots \ldots \\
\text { jéjunum } \ldots \ldots \ldots \ldots \\
\text { iléon } \ldots \ldots \ldots \ldots \ldots\end{array}$ & $\begin{aligned} & 4,0(g h) \\
\pm & 0,7 \\
& 6,2\left({ }^{e f}\right) \\
\pm & 0,8 \\
& 3,9\left({ }^{h i}\right) \\
\pm & 0,4\end{aligned}$ & $\begin{aligned} & 4,8(f g) \\
\pm & 0,4 \\
& 8,0(d e) \\
\pm & 0,8 \\
& 3,0\left({ }^{i j}\right) \\
\pm & 0,7\end{aligned}$ & $\begin{aligned} & 6,5\left({ }^{e f}\right) \\
\pm & 1,1 \\
& 10,4(b) \\
\pm & 1,7 \\
& 2,2(j) \\
\pm & 0,4\end{aligned}$ & 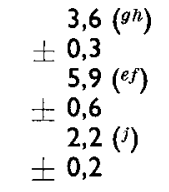 \\
\hline
\end{tabular}

* Les valeurs qui ne sonł pas suivies de la même lettre diffèrent significativement entre elles $(P<0,05)$.

C) Comparaison entre les actions stimulantes des 2 glucides sur les activités maltase ef invertase (tabl. 3).

TABLEAU 3

Valeurs des activités disaccharidases dans le régime saccharose exprimées pour chaque segment en pourcentage des activités mesurées dans le régime amidon

\begin{tabular}{|c|c|c|c|c|c|c|}
\hline \multirow{3}{*}{ Enzymes } & \multicolumn{6}{|c|}{ Poulets de } \\
\hline & \multicolumn{3}{|c|}{4 semaines } & \multicolumn{3}{|c|}{8 semaines } \\
\hline & $D^{*}$ & J & 1 & $\mathrm{D}$ & J & 1 \\
\hline $\begin{array}{l}\text { Maltase } . \ldots \ldots \ldots \ldots \ldots \\
\text { Invertase } \ldots \ldots \ldots \ldots \ldots\end{array}$ & $\begin{array}{l}136 \\
175\end{array}$ & $\begin{array}{l}107 \\
128\end{array}$ & $\begin{array}{l}71 \\
74\end{array}$ & $\begin{array}{r}99 \\
135\end{array}$ & $\begin{array}{l}116 \\
130\end{array}$ & $\begin{array}{l}45 \\
73\end{array}$ \\
\hline
\end{tabular}

* $\mathrm{D}=$ duodénum ; $\mathrm{J}=$ jéjunum ; I == iléon. 
Nous avons constaté que le saccharose et l'amidon stimulent tous les 2 la maltase et l'invertase. On peut se demander s'il n'y a pas quelques différences dans ces actions stimulantes, et si, dans une certaine mesure, le substrat glucidique n'oriente pas les activités enzymatiques. Pour le savoir, nous exprimons les activités trouvées avec le régime saccharose en pourcentage de celles mesurées avec le régime amidon (cf. tabl. 3). Nous vérifions la stimulation inégale induite par les glucides sur les parties proximales et distales de l'intestin : valeurs supérieures à 100 indiquant un effet plus marqué du saccharose (duodénum et jéjunum), valeurs inférieures à 100 pour une action dominante de l'amidon (iléon). D'une manière générale, à chaque âge et dans chaque segment les valeurs trouvées pour l'invertase sont supérieures à celles obtenues pour la maltase. Cela est particulièrement net pour l'iléon à l'âge de 3 semaines. Cependant, aucune différence n'atteint le seuil de signification. Autrement dit, si le saccharose stimule un peu plus l'invertase et par voie de réciprocité si l'amidon augmente surtout l'activité maltase la différence est trop faible ef inconsistante pour faire apparaître une réponse spécifique à chacun des sucres dans nos conditions expérimentales (6 poulets par lot à l'âge de 8 semaines).

\section{Discussion.}

Chez le poulet les activités maltase et invertase varient au long de l'intestin grêle. A la suite d'autres auteurs, nous constatons qu'elles sont généralement maximum au niveau du jéjunum (Levin, 1976).

Les régimes riches en saccharose ef en amidon stimulent conjointement les 2 types d'activité, surtout dans les parties proximales pour le saccharose, dans les parties terminales pour l'amidon. Le devenir des 2 glucides et leur transit avant absorption peut expliquer ce résultat. Soluble et immédiatement disponible le saccharose est rapidement assimilé. En revanche, l'amidon exige l'action préalable de l'amylase pancréatique ; une bonne part du maltose néoformé est absorbé et hydrolysé dans l'iléon. Ainsi, comme chez les mammifères les entérocytes seraient directement influencés par les apports alimentaires (Rosensweig et Herman, 1970 ; Castro et al., 1975), saccharose et maltose stimulant les activités disaccharidases sur les lieux d'absorption.

L'influence de l'âge montre une fois de plus l'existence d'une maturation de l'intestin au cours du développement (Dautlick et Strittmatter, 1970). Rapportées aux poids frais les activités enzymatiques diminueraient systématiquement de 4 à 8 semaines du fait de l'épaisseur accrue de la paroi intestinale. Le mode d'expression choisi dans cette éfude amenuise les diminutions et les rend non significatives dans le cas du régime « commercial ». Chez les poulets sur régimes synthétiques riches en glucides les réductions d'activités sont d'autant plus marquées que la stimulation apparaissait forte à 4 semaines. Dans le même temps les activités tendent à s'accroître chez les poulets ne disposant pas de glucides (graisse-cell). En bref, l'effet des régimes semble quelque peu s'atténuer avec l'âge au profit d'une régulation intrinsèque à l'animal.

L'absence de spécificité ou en tout cas la faible spécificité de la stimulation observée chez le poulet oppose quelque peu cette espèce et les mammifères (Rosensweig et Herman, 1970). Pour expliquer cette absence de spécificité on pourrait invoquer l'existence d'une protéine unique porteuse des 2 activités. Maltase et invertase sont, il est vrai, 
souvent assemblés sur les mêmes complexes protidiques, mais leur apparition après la naissance dans la bordure en brosse de l'intestin est observée à des âges différents (Raul et al., 1978). En outre, l'invertase a pu être isolée sous une forme ne comportant que 2 activités, invertase ef isomaltase, cela aussi bien chez le poulet (Siddons, 1970) que chez les mammifères (Nishi et Takesue, 1978).

En définitive, l'existence de plusieurs enzymes à activités maltase et invertase parfois associées (Asp et al., 1974) peut rendre comple des différences entre espèces. On peut aussi invoquer une action inégale des produits de dégradation : glucose et fructose. Ces derniers, capables d'accroître les activités disaccharidases par voie parentérale, seraient en effet les agents actifs de la stimulation par les glucides alimentaires (Rosensweig ef Herman, 1970 ; Phan ef Le Van Hung, 1975). L'absence de spécificité chez les oiseaux pourrait tenir à une plus faible activité du fructose ou à une action indifférente des 2 oses.

Commission CNERNA Digestion-Absorption/Association des Physiologistes, Paris 5-6 octobre 1978.

\section{Références}

ASP N. G., GUTMAD-HÖYER P. M., CHRISTIANSEN P. M., DAHLQUIST A., $1974 . \quad$ Acid $\alpha$ glucosidase from human gastrointestinal mucosa. Separation and characterization. Scand $\mathrm{J}$. clin. lab. Invest., 33, 239-245.

CASTRO G. A., LOPELAND E. M., DUDRICK S. J., JOHNSON L. R., 1975. Intestinal disaccharidase and peroxydase activities in parenterally nourished rats. J. Nutr., 105, 776-781.

DAHLQUIST A., 1964. Method for assay of intestinal disaccharidases. Anal. Biochem., 7, 18-25.

DAUTLICK J., STRITTMATTER C. F., 1970. Developmental and hormone-induced changes in chicken intestinal disaccharidases. Biochim. biophys. Acto, 222, 444-454.

GAUTHIER A., LARBIER M., BLUM J. C., 1979. Influence des glucides alimentaires sur la croissance ef la vitesse d'absorption intestinale du glucose el du fructose chez le poulet. Ann. Biol. anim. Biochim. Biophys., 19, 703-707.

LEVIN R. J., 1976. Digestion and absorption of carbohydrate from embryo to adult, 63-116. In BOORMAN K. N., FREEMAN B. M., Digestion in the fowl. Brit. Poult. Sci. Ltd, Edinburg.

LOWRY O. H., ROSEBROUGH N. J., FARR A. L., RANDALL R. J., 1951. Protein measurement with the folin reagent. J. biol. Chem., 133, 265-275.

NISHI Y., TAKESUE Y., 1978. Electron microscope studies on triton solubilized sucrase from rabbit small intestine. J. Ultrastruct. Res., 62, 1-12.

PHAN H. H., LE VAN HUNG, 1975. Réponses de la saccharase intestinale et modifications physiologiques observées chez le rat ayant ingéré des régimes à différents constituants glucidiquas. Ann. Nutr. Alim., 29, 395-410.

RAUL F., SIMON P. M., KEDINGER M., GRENIER J. F., HAFFEN K., 1978. Separation and characterization of intestinal brush border enzymes in adult rats and in suckling rat under conditions and after hydrocortisone injections. Enzyme, 23, 89-97.

ROSENSWEIG N. S., HERMAN R. H., 1970. Dose response of jejunal sucrase and maltase activities to isocaloric high and low carbohydrate diets in man. Amer. J. clin. Nutr., 23, 1373-1377.

SIDDONS R. C., 1970. Heat inactivation and Sephadex chromatography of the small intestine disaccharidases of the chick. Biochem. J., 166, 71-78.

SIDDONS R. C., 1972. Effect of diet on disaccharidase activity in the chick. Br. J. Nutr., 27, 343-352. 